\title{
TAMENESS OF PAIRS OF NUCLEAR POWER SERIES SPACES AND RELATED TOPICS
}

BY

\author{
KAISA NYBERG
}

\begin{abstract}
The equivalence of the following six assertions is proved: (i) The set of the finite limit points of the ratios $\alpha_{m} / \beta_{n}, n, m \in \mathbf{N}$, is bounded. (ii) Every operator from $\Lambda_{\infty}(\beta)$ to $\Lambda_{1}(\alpha)$ is compact. (iii) The pair $\left(\Lambda_{\infty}(\beta), \Lambda_{1}(\alpha)\right)$ is tame, i.e., for every operator $T$ from $\Lambda_{\infty}(\beta)$ to $\Lambda_{1}(\alpha)$ there is a positive integer $a$ such that for every $k \in \mathbf{N}$ there is a constant $C_{k}$ such that $\|T x\|_{k} \leqslant C_{k}|x|_{a k}$ for every $x \in \Lambda_{\infty}(\beta)$. (iv) Every short exact sequence $0 \rightarrow \Lambda_{\tau}(\beta) \rightarrow X \rightarrow \Lambda_{1}(\alpha) \rightarrow 0$, where $\tau=1$ or $\infty$, splits. (v) $\Lambda_{1}(\alpha) \times \Lambda_{\infty}(\beta)$ has a regular basis. (vi) $\Lambda_{1}(\alpha) \otimes \Lambda_{\infty}(\beta)$ has a regular basis. Also the finite type power series spaces that contain subspaces isomorphic to an infinite type power series space are characterized as well as the infinite type power series spaces that have finite type quotient spaces.
\end{abstract}

Introduction. In the theory of nuclear Fréchet spaces, the power series spaces provide natural examples and their study has greatly contributed to the development of the general theory. During the past fifteen years several authors have considered various relations between two power series spaces. The purpose of this paper is to give a unifying treatment of some of the relations between two power series spaces of different type and at the same time fill in the gaps in the earlier studies.

Given a power series spaces of finite type $\Lambda_{1}(\alpha)$ and a second one of infinite type $\Lambda_{\infty}(\beta)$, it may happen that all the operators from $\Lambda_{\infty}(\beta)$ to $\Lambda_{1}(\alpha)$ are compact, but usually this is not the case (see $[2,3,6,7,9,13,14,17$ and 18$]$ ). In the literature [10, 12, and 16] a few examples have been given of short exact sequences $0 \rightarrow \Lambda_{\infty}(\beta) \rightarrow$ $X \rightarrow \Lambda_{1}(\alpha) \rightarrow 0$ and $0 \rightarrow \Lambda_{1}(\beta) \rightarrow X \rightarrow \Lambda_{1}(\alpha) \rightarrow 0$ that do not split. In [10] it was shown that usually this type of nonsplitting short exact sequences exist and can also be constructed. Now we shall see that this is the case exactly when the set of the finite limit points of the ratios $\alpha_{m} / \beta_{n}$ is unbounded. This condition also determines whether there exist noncompact operators from $\Lambda_{\infty}(\beta)$ to $\Lambda_{1}(\alpha)$. Moreover, the condition given in [1], under which there exist regular bases in the product spaces $\Lambda_{1}(\alpha) \times \Lambda_{\infty}(\beta)$ and $\Lambda_{1}(\alpha) \otimes \Lambda_{\infty}(\beta)$, turns out to be just another way of saying that the set of the finite limit points of the set $\left\{\alpha_{m} / \beta_{n} \mid m, n \in \mathbf{N}\right\}$ is bounded.

In the first section we study the tameness of pairs of Fréchet spaces. The concept of tameness is due to E. Dubinsky and its introduction was motivated by the following fact: In a tame infinite type power series space every complemented subspaces has a basis. (This unpublished result of Dubinsky and D. Vogt has been privately communicated to the author by Dubinsky.) The characterization of the

Received by the editors May 23, 1983.

1980 Mathematics Subject Classification. Primary 46A12. 
tameness of the pair $\left(\Lambda_{\infty}(\beta), \Lambda_{1}(\alpha)\right)$ is where we come out with the crucial condition. The splitting of short exact sequences and the existence of regular bases in product spaces are discussed in $\S \S 4$ and 5 . The existence of embeddings from a coordinate subspace of $\Lambda_{\infty}(\beta)$ to $\Lambda_{1}(\alpha)$ is closely related to the existence of noncompact operators. These are not, however, equivalent questions, as we show in $\S 2$, where we also correct a mistake in [6] and settle the problems presented in [2]. In $\$ 3$ the corresponding questions are considered about quotient mappings and a necessary and sufficient condition is given for an infinite type power series space to have a finite type quotient. An extensive study of isomorphic embeddings and compact operators in different types of generalized power series spaces was given by V. V. Kashirin in [7 and 9]; some of these results can be found in [8]. Our results in $\$ 2$ are special cases of Kashirin's general theorems although our conditions look different.

At this point I would like to express my gratitude to Professor Ed Dubinsky for drawing my attention to the tameness of the cartesian product $\Lambda_{1}(\alpha) \times \Lambda_{\infty}(\beta)$, as well as his valuable comments. I would also like to thank Professor James E. West for his hospitality and interest in my work during my stay at Cornell University.

1. Tameness. Throughout this section we shall consider Fréchet spaces $E$ and $F$ with fixed sequences of continuous seminorms $\left(|\cdot|_{k}\right)$ and $\left(\|\cdot\|_{k}\right)$.

1.1. Definition. The pair $(E, F)$ is tame if for every operator $T: E \rightarrow F$ there is an integer $a>0$ such that

$$
\sup _{x \in E} \frac{\|T x\|_{k}}{|x|_{a k}}<\infty
$$

for every $k \in \mathbf{N}$. The pair $(E, F)$ is said to be tameable if there exist systems of continuous seminorms in $E$ and $F$ with respect to which the pair $(E, F)$ is tame. If the pair $(E, E)$ is tame (tameable) we say that the space $E$ is tame (tameable).

Notice that the pair $(E, F)$ is tameable if and only if there exists an increasing function $\sigma: \mathbf{N} \rightarrow \mathbf{N}$ such that

$$
\sup _{x \in E} \frac{\|T x\|_{k}}{|x|_{\sigma(k)}}<\infty
$$

for every operator $T: E \rightarrow F$ and for every $k$ sufficiently large.

The nuclear Köthe spaces $K(a), a=\left(a_{n}^{k}\right)$ can be equipped with two equivalent systems of continuous seminorms:

$$
x=\left(x_{n}\right) \mapsto \sum_{n=1}^{\infty}\left|x_{n}\right| a_{n}^{k}, \quad k \in \mathbf{N},
$$

or

$$
x=\left(x_{n}\right) \mapsto \sup _{n \in \mathbf{N}}\left|x_{n}\right| a_{n}^{k}, \quad k \in \mathbf{N},
$$

which we will refer to as sum-seminorms and sup-seminorms respectively.

If we have the sum-seminorms in $E=K(a)$ then the supremums in (1) and (2) will be finite if they are finite taken over the coordinate basis vectors $x=e_{n}, n \in \mathbf{N}$, only. 
Power series spaces $\Lambda_{1}(\alpha)$ of finite type and $\Lambda_{\infty}(\alpha)$ of infinite type are the Köthe spaces defined by the matrices $a=\left(a_{n}^{k}\right)$.

$$
a_{n}^{k}=e^{-\alpha_{n} / k} \quad \text { and } \quad a_{n}^{k}=e^{k \alpha_{n}},
$$

respectively, where the exponent sequence $\alpha=\left(\alpha_{n}\right)$ is an increasing sequence of positive numbers with $\lim _{n \rightarrow \infty} \alpha_{n}=\infty$. For the nuclearity of power series spaces and other concepts that remain undefined, we refer to [5]. Throughout this paper all power series spaces will be assumed to be nuclear.

For any nuclear power series space there exists an integer $l$ such that every $k$ th sum-norm is dominated by the $(k+l)$ th sup-norm. Since for every $a \geqslant 1$ there is $b \geqslant a$ such that $a k+l \leqslant a(k+l) \leqslant b k$, the tameness of a pair of power series spaces does not depend on which of the natural systems of seminorms, sums or supremums, is used. For this reason the statements of the propositions below will be valid for both norm-systems. In the proofs we shall use the most convenient ones.

The following fact discovered by Dubinsky is implicitly present already in [11].

1.2. Proposition. Pairs of nuclear finite type power series spaces are tame.

Proof. Let $|\cdot|_{k}, k \in \mathbf{N}$, be the sum-norms in $\Lambda_{1}(\beta)$ and $\|\cdot\|_{k}, k \in \mathbf{N}$, the sup-norms in $\Lambda_{1}(\alpha)$ and consider an operator $T: \Lambda_{1}(\beta) \rightarrow \Lambda_{1}(\alpha)$. Let $\sigma$ be a function $\mathbf{N} \rightarrow \mathbf{N}$ such that

$$
\sup _{n} \frac{\left\|T e_{n}\right\|_{k}}{\left|e_{n}\right|_{\sigma(k)}}=C_{k}<\infty, \quad k \in \mathbf{N},
$$

where $\left(e_{n}\right)$ is the coordinate basis. We may assume the sequence of constants $\left(C_{k}\right)$ is increasing. Write

$$
T e_{n}=\sum_{j=1}^{\infty} \lambda_{j}^{n} e_{j}
$$

Then there are indices $q_{n}^{k}$ such that

$$
\left\|T e_{n}\right\|_{k}=\sup _{j}\left|\lambda_{j}^{n}\right| \exp \left(-\frac{1}{k} \alpha_{j}\right)=\left|\lambda_{q_{n}^{k}}\right| \exp \left(-\frac{1}{k} \alpha_{q_{n}^{k}}\right), \quad k, n \in \mathbf{N} .
$$

Then

$$
\exp \left\{\left(\frac{1}{l}-\frac{1}{k}\right) \alpha_{q_{n}^{\prime}}\right\} \leqslant \frac{\left\|T e_{n}\right\|_{k}}{\left\|T e_{n}\right\|_{l}} \leqslant \exp \left\{\left(\frac{1}{l}-\frac{1}{k}\right) \alpha_{q_{n}^{k}}\right\}, \quad n, k \in \mathbf{N} .
$$

Consequently, for any $a>0$ :

$$
\begin{aligned}
C_{k} & \geqslant \frac{\left\|T e_{n}\right\|_{k}}{\left|e_{n}\right|_{\sigma(k)}}=\frac{\left\|T e_{n}\right\|_{k}}{\left\|T e_{n}\right\|_{l}} \frac{\left\|T e_{n}\right\|_{l}}{\left|e_{n}\right|_{a l}} \frac{\left|e_{n}\right|_{a l}}{\left|e_{n}\right|_{\sigma(k)}} \\
& \geqslant \exp \left\{\left(\frac{1}{l}-\frac{1}{k}\right) \alpha_{q_{n}^{\prime}}\right\} \exp \left\{\left(\frac{1}{\sigma(k)}-\frac{1}{a l}\right) \beta_{n}\right\} \frac{\left\|T e_{n}\right\|_{l}}{\left|e_{n}\right|_{a l}} .
\end{aligned}
$$


So it is enough to show that there is $a \geqslant 1$ such that for every $l$ there is $k$ such that

$$
\left(\frac{1}{l}-\frac{1}{k}\right) \alpha_{q_{n}^{\prime}}+\left(\frac{1}{\sigma(k)}-\frac{1}{a l}\right) \beta_{n} \geqslant 0
$$

or, equivalently,

$$
\frac{1}{l}\left(\alpha_{q_{n}^{\prime}}-\frac{1}{a} \beta_{n}\right) \geqslant \frac{1}{k}\left(\alpha_{q_{n}^{\prime}}-\frac{k}{\sigma(k)} \beta_{n}\right) .
$$

We choose $a=2 \sigma(1)$ and consider two cases. First,

$$
\alpha_{q_{n}^{\prime}} \leqslant(2 / a) \beta_{n} .
$$

Then

$$
\frac{1}{l}\left(\alpha_{q_{n}^{\prime}}-\frac{1}{a} \beta_{n}\right) \geqslant \frac{1}{l}\left(\alpha_{q_{n}^{\prime}}-\frac{2}{a} \beta_{n}\right) \geqslant \alpha_{q_{n}^{\prime}}-\frac{2}{a} \beta_{n}=\alpha_{q_{n}^{\prime}}-\frac{1}{\sigma(1)} \beta_{n} .
$$

So for these $n$ choose $k=1$. Secondly,

$$
\alpha_{q_{n}^{l}}>(2 / a) \beta_{n} .
$$

Then

$$
\begin{aligned}
\frac{1}{l}\left(\alpha_{q_{n}^{\prime}}-\frac{1}{a} \beta_{n}\right) & =\frac{1}{2 l} \alpha_{q_{n}^{\prime}}+\frac{1}{2 l} \alpha_{q_{n}^{\prime}}-\frac{1}{a l} \beta_{n} \\
& \geqslant \frac{1}{2 l} \alpha_{q_{n}^{\prime}} \geqslant \frac{1}{2 l}\left(\alpha_{q_{n}^{\prime}}-\frac{2 l}{\sigma(2 l)} \beta_{n}\right) .
\end{aligned}
$$

So $k=2 l$ works for these $n$. Now it follows from (3) that

$$
\left\|T e_{n}\right\|_{l} \leqslant C_{2 l}\left\|e_{n}\right\|_{2 \sigma(1) \cdot l}, \quad n \in \mathbf{N} .
$$

An operator $T: E \rightarrow F$ is compact if there is a zero-neighborhood $U$ in $E$ such that $T(U)$ is relatively compact in $F$. In a nuclear Fréchet space bounded sets are relatively compact, so assuming the nuclearity of $E$ and $F$, an operator $T: E \rightarrow F$ is compact if and only if there is an $l$ such that for every $k$,

$$
\sup _{x \in E} \frac{\|T x\|_{k}}{|x|_{l}}<\infty .
$$

According to Zaharjuta [18] all operators $T: \Lambda_{1}(\beta) \rightarrow \Lambda_{\infty}(\alpha)$ are compact. So we have the following.

1.3. Proposition. Any pair $\left(\Lambda_{1}(\beta), \Lambda_{\infty}(\alpha)\right)$ is tame.

More interesting is the situation for pairs $\left(\Lambda_{\infty}(\beta), \Lambda_{1}(\alpha)\right)$ and $\left(\Lambda_{\infty}(\beta), \Lambda_{\infty}(\alpha)\right)$. The tameness of an infinite type power series space has been studied by Dubinsky and Vogt. Their results can be extended to the case of different exponent sequences and we state the following proposition without proof. We use the notation $\operatorname{LIM}\{\alpha / \beta\}$ for the set of the finite limit points of the set $\left\{\alpha_{m} / \beta_{n} \mid m, n \in \mathbf{N}\right\}$.

1.4. Proposition. The following three conditions are equivalent.

(i) The pair $\left(\Lambda_{\infty}(\beta), \Lambda_{\infty}(\alpha)\right)$ is tame.

(ii) The pair $\left(\Lambda_{\infty}(\beta), \Lambda_{\infty}(\alpha)\right)$ is tameable.

(iii) The set $\operatorname{LIM}\{\alpha / \beta\}$ is bounded. 
Next we shall study in detail the tameness of the pair $\left(\Lambda_{\infty}(\beta), \Lambda_{1}(\alpha)\right)$. The following proposition was first proved by V. V. Kashirin [6] as an application of a lemma of M. M. Dragilev [3].

1.5. Proposition. If $\operatorname{LIM}\{\alpha / \beta\}$ is bounded then every operator from $\Lambda_{\infty}(\beta)$ to $\Lambda_{1}(\alpha)$ is compact.

Proof. Let $T: \Lambda_{\infty}(\beta) \rightarrow \Lambda_{1}(\alpha), T e_{n}=\sum_{j=1}^{\infty} t_{j}^{n} e_{j}$, be a continuous operator; so there is an increasing map $\sigma: \mathbf{N} \rightarrow \mathbf{N}$ and positive constants $C_{k}, k \in \mathbf{N}$, such that

$$
\|T x\|_{k} \leqslant C_{k}|x|_{\sigma(k)}, \quad x \in \Lambda_{\infty}(\beta), k \in \mathbf{N} .
$$

Let $q_{n}^{k}$ be the index on which the $k$ th seminorm of $T e_{n}$ attains its value; then for every $k$ and $l$ we have Dubinsky's inequality:

$$
\exp \left\{\left(\frac{1}{k}-\frac{1}{l}\right) \alpha_{q_{n}^{k}}\right\} \leqslant \frac{\left\|T e_{n}\right\|_{l}}{\left\|T e_{n}\right\|_{k}} \leqslant \exp \left\{\left(\frac{1}{k}-\frac{1}{l}\right) \alpha_{q_{n}^{\prime}}\right\} .
$$

From this and (4) it then follows that for every $k, l, p$ and $n$ we have

$$
\begin{aligned}
C_{l} & \geqslant \frac{\left\|T e_{n}\right\|_{l}}{\left|e_{n}\right|_{\sigma(l)}}=\frac{\left\|T e_{n}\right\|_{l}}{\left\|T e_{n}\right\|_{k}} \cdot \frac{\left\|T e_{n}\right\|_{k}}{\left|e_{n}\right|_{p}} \cdot \frac{\left|e_{n}\right|_{p}}{\left|e_{n}\right|_{\sigma(l)}} \\
& \geqslant \exp \left\{\left(\frac{1}{k}-\frac{1}{l}\right) \alpha_{q_{n}^{k}}-(\sigma(l)-p) \beta_{n}\right\} \cdot \frac{\left\|T e_{n}\right\|_{k}}{\left|e_{n}\right|_{p}} .
\end{aligned}
$$

Let $B>0$ be a strict upper bound of $\operatorname{LIM}\{\alpha / \beta\}$, and let $k$ and $n$ be fixed. Then there are three possibilities.

(i) $\alpha_{q_{n}^{k}} / \beta_{n} \leqslant B$. Choose $l=1, p=B+\sigma(1)$; then

$$
\left(\frac{1}{k}-\frac{1}{l}\right) \alpha_{q_{n}^{k}}-(\sigma(l)-p) \beta_{n} \geqslant \beta_{n}\left(-\frac{\alpha_{q_{n}^{k}}}{\beta_{n}}+B\right) \geqslant 0 .
$$

So, by (6) we have, in this case,

$$
\left\|T e_{n}\right\|_{k} \leqslant C_{1}\left|e_{n}\right|_{B+\sigma(1)} .
$$

(ii) $\alpha_{q_{n}^{k}} / \beta_{n} \geqslant k(k+1) \sigma(k+1)$. Now

$$
\left(\frac{1}{k}-\frac{1}{k+1}\right) \alpha_{q_{n}^{k}}-(\sigma(k+1)-1) \beta_{n} \geqslant \beta_{n}>0 ;
$$

so we get from (6) that

$$
\left\|T e_{n}\right\|_{k} \leqslant C_{k+1}\left|e_{n}\right|_{1} .
$$

(iii) It follows from our assumptions that for a fixed $k$ the remaining case can happen only for finite number of indices $n$. Combining (i)-(iii) we obtain

$$
\sup _{n \in \mathbf{N}} \frac{\left\|T e_{n}\right\|_{k}}{\left|e_{n}\right|_{B+\sigma(1)}}<\infty, \quad k \in \mathbf{N}
$$

therefore $T$ is compact.

1.6. Proposition. If $\operatorname{LIM}\{\alpha / \beta\}$ is unbounded then the pair $\left(\Lambda_{\infty}(\beta), \Lambda_{1}(\alpha)\right)$ is not tameable. 
Proof. For a given $\sigma: \mathbf{N} \rightarrow \mathbf{N}$ we shall construct a continuous operator $T$ : $\Lambda_{\infty}(\beta) \rightarrow \Lambda_{1}(\alpha)$ such that

$$
\sup _{n \in \mathbf{N}} \frac{\left\|T e_{n}\right\|_{k}}{\left|e_{n}\right|_{\sigma(k)}}=\infty, \quad \text { for all } k \in \mathbf{N} .
$$

Let $r_{1} \in \operatorname{LIM}\{\alpha / \beta\}$ be positive and $N_{1} \subset \mathbf{N}$ such that

$$
\lim _{\substack{n \in N_{1} \\ n \rightarrow \infty}} \frac{\alpha_{m(1, n)}}{\beta_{n}}=r_{1} .
$$

Split $N_{1}$ into two disjoint infinite subsets $N_{1}^{1}$ and $N_{1}^{2}$. Then for one of the sets, $\mathbf{N} \backslash N_{1}, N_{1}^{1}$ or $N_{1}^{2}$, call it $L$, the set of the finite limit points of $\left\{\alpha_{m} / \beta_{n} \mid m \in \mathbf{N}\right.$, $n \in L\}$ is unbounded. If $L=\mathbf{N} \backslash N_{1}$, choose $M_{1}=N_{1}$; if $L=N_{1}^{1}$ (or $L=N_{1}^{2}$ ) choose $M_{1}=N_{1}^{2}$ (or $M_{1}=N_{1}^{1}$ respectively). In all cases (7) remains true if $N_{1}$ is replaced by $M_{1}$. Let $r_{2}>r_{1}+1$ be a limit point of $\left\{\alpha_{m} / \beta_{n} \mid m \in \mathbf{N}, n \in \mathbf{N} \backslash M_{1}\right\}$ and repeat the above step for $\mathbf{N} \backslash M_{1}$ and $r_{2}$ instead of $\mathbf{N}$ and $r_{1}$. In this manner, inductively, we obtain a sequence of limit points $\left(r_{k}\right), \lim r_{k}=\infty$, and disjoint $M_{k}$, such that there are indices $m(k, n)$ with

$$
\lim _{\substack{n \in M_{k} \\ n \rightarrow \infty}} \frac{\alpha_{m(k, n)}}{\beta_{n}}=r_{k}
$$

Denote by $I_{k}$ the open interval

$$
(k(k-1)(\sigma(k+1)-\sigma(k)), k(k+1)(\sigma(k+2)-\sigma(k+1))) .
$$

Increasing $\sigma$ we may assume there is at least one limit point $r_{k}$ on $I_{k}$ and

$$
\sigma(k+1)-\sigma(k) \geqslant \sigma(k)-\sigma(k-1)>0 \text { for all } k \in \mathbf{N} .
$$

Passing to a subsequence of $\left(r_{k}\right)$ and discarding finitely many indices in each $M_{k}$, $k \in \mathbf{N}$, we have disjoint infinite sets $M_{k}$ and indices $m(k, n), n \in M_{k}, k=1,2, \ldots$, such that

$$
\alpha_{m(k, n)} / \beta_{n} \in I_{k}, \quad n \in M_{k}, k=1,2, \ldots
$$

Now we define

$$
T e_{n}=\left\{\begin{array}{l}
\exp \left\{\sigma(k+1) \beta_{n}+\frac{1}{k} \alpha_{m(k, n)}\right\} e_{m(k, n)}, \quad n \in M_{k}, k=1,2, \ldots, \\
0, \quad n \notin \bigcup_{k=1}^{\infty} M_{k} .
\end{array}\right.
$$

Then

$$
\left\|T e_{n}\right\|_{k}=e^{\sigma(k+1) \beta_{n}}=\left|e_{n}\right|_{\sigma(k+1)}, \quad n \in M_{k}, k=1,2, \ldots
$$

Next we prove by induction that for any $l$,

$$
\left\|T e_{n}\right\|_{l} \leqslant\left|e_{n}\right|_{\sigma(l+1)}, \quad n \in \cup M_{k} .
$$

Fix $k \in \mathbf{N}$. Since $\alpha_{m(k, n)} \geqslant k(k-1)(\sigma(k+1)-\sigma(k)) \beta_{n}$, it follows that

$$
\left\|T e_{n}\right\|_{k-1}=\exp \left\{\sigma(k+1) \beta_{n}+\left(\frac{1}{k}-\frac{1}{k-1}\right) \alpha_{m(k, n)}\right\} \leqslant e^{\sigma(k) \beta_{n}} .
$$


Now assume the claim is true for for $l=k-j>1$. Then

$$
\begin{aligned}
\left\|T e_{n}\right\|_{l-1} & =\exp \left\{\sigma(k+1) \beta_{n}+\left(\frac{1}{k}-\frac{1}{l}\right) \alpha_{m(k, n)}+\left(\frac{1}{l}-\frac{1}{l-1}\right) \alpha_{m(k, n)}\right\} \\
& =\left\|T e_{n}\right\|_{l} \exp \left\{\left(\frac{1}{l}-\frac{1}{l-1}\right) \alpha_{m(k, n)}\right\} \leqslant e^{\sigma(l) \beta_{n}},
\end{aligned}
$$

since

$$
\alpha_{m(k, n)} \geqslant k(k-1)(\sigma(k+1)-\sigma(k)) \beta_{n} \geqslant l(l-1)(\sigma(l+1)-\sigma(l)) \beta_{n}
$$

and

$$
\left\|T e_{n}\right\|_{l} \leqslant e^{\sigma(l+1) \beta_{n}} .
$$

So we have proved that

$$
\left\|T e_{n}\right\|_{l} \leqslant\left|e_{n}\right|_{\sigma(l+1)} \text { for } l \leqslant k .
$$

For seminorms $l>k$ the proof is similar. Thus it follows from the construction of $T$ that it is a continuous operator from $\Lambda_{\infty}(\beta)$ to $\Lambda_{1}(\alpha)$ and

$$
\sup _{n \in \mathbf{N}} \frac{\left\|T e_{n}\right\|_{k}}{\left|e_{n}\right|_{\sigma(k)}} \geqslant \sup _{n \in M_{k}} \frac{\left\|T e_{n}\right\|_{k}}{\left|e_{n}\right|_{\sigma(k)}}=\infty \text {. }
$$

Combining Propositions 1.5 and 1.6 we get

1.7. THEOREM. The following four conditions are equivalent.

(i) Every operator from $\Lambda_{\infty}(\beta)$ to $\Lambda_{1}(\alpha)$ is compact.

(ii) The pair $\left(\Lambda_{\infty}(\beta), \Lambda_{1}(\alpha)\right)$ is tame.

(iii) The pair $\left(\Lambda_{\infty}(\beta), \Lambda_{1}(\alpha)\right)$ is tameable.

(iv) The set $\operatorname{LIM}\{\alpha / \beta\}$ is bounded.

Notice that each of these conditions is equivalent to those in Proposition 1.4. Let us also remark that the equivalence (i) $\Leftrightarrow$ (iv) could also be proven using the general theorems of Vogt [17].

An earlier characterization of the pairs of exponent sequences $\alpha$ and $\beta$ for which every operator from $\Lambda_{\infty}(\alpha)$ to $\Lambda_{1}(\beta)$ is compact was given by N. DeGrandeDeKimpe and W. B. Robinson in [2]. So their condition (a) of Lemma 3.1 in [2] is equivalent to the boundedness of the set $\operatorname{LIM}\{\beta / \alpha\}$ and their condition (b) of the same lemma is just a more technical way of saying that $\operatorname{LIM}\{\beta / \alpha\}$ is unbounded.

An operator $T: \Lambda_{1}(\alpha) \times \Lambda_{\infty}(\beta) \rightarrow \Lambda_{1}(\alpha) \times \Lambda_{\infty}(\beta)$ has a matrix presentation

$$
T=\left(\begin{array}{ll}
T_{11} & T_{12} \\
T_{21} & T_{22}
\end{array}\right):(x, y) \mapsto\left(T_{11} x+T_{12} y, T_{21} x+T_{22} y\right) .
$$

So in view of Propositions 1.2 and 1.3 the space $\Lambda_{1}(\alpha) \times \Lambda_{\infty}(\beta)$ is tame (or tameable) if and only if $\Lambda_{\infty}(\beta)$ and the pair $\left(\Lambda_{\infty}(\beta), \Lambda_{1}(\alpha)\right)$ are tame. This observation combined with Theorems 1.4 and 1.7 gives us the following

1.8. THEOREM. The cartesian product $\Lambda_{1}(\alpha) \times \Lambda_{\infty}(\beta)$ is tame if and only if $\operatorname{LIM}\{\beta / \beta\}$ and $\operatorname{LIM}\{\alpha / \beta\}$ are bounded. 
2. Infinite type subspaces of finite type power series spaces. This topic has previously been studied by Dubinsky [4], Kashirin [6, 7] and DeGrande-DeKimpe and Robinson [2]. Kashirin gave a characterization of those finite type power series spaces that contain subspaces isomorphic to infinite type power series spaces. Unfortunately there is a serious misprint in the statement of his theorem in [6]. It essentially weakens the given condition so that it is impossible to make the embedding for which he refers to Dubinsky [4]. DeGrande-DeKimpe and Robinson studied compact operators from $\Lambda_{\infty}(\beta)$ to $\Lambda_{1}(\alpha)$, but did not completely determine the cases when $\Lambda_{1}(\alpha)$ contains subspaces isomorphic to some $\Lambda_{\infty}(\beta)$. The following theorem can be stated.

2.1. THEOREM. The following three conditions are equivalent.

(i) $\Lambda_{\infty}(\beta)$ contains a coordinate subspace isomorphic to a subspace of $\Lambda_{1}(\alpha)$.

(ii) There is a subset $M \subset \mathbf{N}$ such that $\operatorname{LIM}\left\{\alpha_{m} / \beta_{n} \mid m \in \mathbf{N}, n \in M_{1}\right\}$ is unbounded for every infinite $M_{1} \subset M$.

(iii) There is a subsequence $\left(\beta_{n_{j}}\right)$ of $\beta$, an increasing sequence $\left(r_{k}\right)$ of positive numbers with $\lim _{k \rightarrow \infty} r_{k}=\infty$, and indices $m(k, j)$ such that $\lim _{j \rightarrow \infty} \alpha_{m(k, j)} / \beta_{n_{j}}=r_{k}$.

Proof. If (ii) is not true, then by Proposition 1.5 every coordinate subspace of $\Lambda_{\infty}(\beta)$ contains a coordinate subspace from which every operator to $\Lambda_{1}(\alpha)$ is compact. Therefore (i) cannot hold. So we have (i) $\Rightarrow$ (ii).

Now assume (ii) holds. Then there are indices $m(1, j)$, a subsequence $(n(1, j))$ of $M$ and a positive limit point $r_{1}$ such that

$$
\lim _{j \rightarrow \infty} \frac{\alpha_{m(1, j)}}{\beta_{n(1, j)}}=r_{1} .
$$

Since the set $\{n(1, j) \mid j \in \mathbf{N}\} \subset \mathbf{N}$ is infinite, it follows from our assumption that there is a second limit point $r_{2} \geqslant r_{1}+1$, indices $m(2, j)$ and a subsequence $\left(\beta_{n(2, j)}\right)$ of $\left(\beta_{n(1, j)}\right)$ such that

$$
\lim _{j \rightarrow \infty} \frac{\alpha_{m(2, j)}}{\beta_{n(2, j)}}=r_{2} .
$$

In this way, by induction, we obtain subsequences $(n(1, j)) \supset(n(2, j)) \supset \cdots$, and we set $n_{j}=n(j, j)$. Then the subsequence $\left(\beta_{n_{j}}\right)$ of $\beta$ satisfies the hypothesis of (iii).

To verify the implication (iii) $\Rightarrow$ (i) we shall, for historical reasons, work a little harder than necessary and show that if (iii) is satisfied then for any given $\sigma: \mathbf{N} \rightarrow \mathbf{N}$ there is an embedding $T$ from a coordinate subspace $\Lambda_{\infty}\left(\beta_{n_{i}}\right)$ of $\Lambda_{\infty}(\beta)$ to $\Lambda_{1}(\alpha)$ such that

$$
\sup _{i \in \mathbf{N}} \frac{\left\|T e_{n_{i}}\right\|_{k}}{\left|e_{n_{i}}\right|_{\sigma(k)}}=\infty
$$

for every $k \in \mathbf{N}$. Now let $\sigma: \mathbf{N} \rightarrow \mathbf{N}$ be given; by increasing it if necessary, we can assume it is strictly increasing and there are indices $q_{j}^{k}, j \geqslant j_{k}, k \in \mathbf{N}$, such that

$$
(k-1) k(\sigma(k+1)-\sigma(k))<\alpha_{q_{j}^{k}} / \beta_{n_{j}}<k(k+1)(\sigma(k+2)-\sigma(k+1)) .
$$


We define

$$
T e_{n_{j}}=\sum_{l=1}^{k} t_{q_{j}^{\prime}} e_{q_{j}^{l}} \text { for } j_{k} \leqslant j<j_{k+1}
$$

where

$$
t_{q_{j}^{l}}=\exp \left\{\sigma(l+1) \beta_{n_{j}}+\frac{1}{l} \alpha_{q_{j}^{l}}\right\}, \quad l=1, \ldots, k, \quad j_{k} \leqslant j<j_{k+1} .
$$

Since

$$
\begin{aligned}
\frac{t_{q_{j}^{l+1}}}{t_{q_{j}^{l}}} & =\exp \left\{(\sigma(l+2)-\sigma(l+1)) \beta_{n_{j}}+\frac{1}{l+1} \alpha_{q_{j}^{l+1}}-\frac{1}{l} \alpha_{q_{j}^{l}}\right\} \\
& \leqslant \exp \left\{\frac{1}{l(l+1)} \alpha_{q_{j}^{l+1}}+\frac{1}{l+1} \alpha_{q_{j}^{l+1}}-\frac{1}{l} \alpha_{q_{j}^{l}}\right\} \\
& =\exp \left\{-\frac{1}{l} \alpha_{q_{j}^{l}}\right\} / \exp \left\{-\frac{1}{l} \alpha_{q_{j}^{l+1}}\right\}
\end{aligned}
$$

and

$$
\begin{aligned}
\frac{t_{q_{j}^{l+1}}}{t_{q_{j}^{l}}} & \geqslant \exp \left\{\frac{1}{l(l+1)} \alpha_{q_{j}^{l}}+\frac{1}{l+1} \alpha_{q_{j}^{l+1}}-\frac{1}{l} \alpha_{q_{j}^{l}}\right\} \\
& =\exp \left\{-\frac{1}{l+1} \alpha_{q_{j}^{l}}\right\} / \exp \left\{-\frac{1}{l+1} \alpha_{q_{j}^{l+1}}\right\},
\end{aligned}
$$

it follows from Dubinsky's lemma (see [5, Proposition II.2.2.3]) that

$$
\left\|T e_{n_{j}}\right\|_{l}=t_{q_{j}^{\prime}} \exp \left\{-\frac{1}{l} \alpha_{q_{j}^{\prime}}\right\}=\exp \left\{\sigma(l+1) \beta_{n_{j}}\right\}=\left|e_{n_{j}}\right|_{\sigma(l+1)}
$$

for $l=1, \ldots, k$ and $j_{k} \leqslant j<j_{k+1}$.

So $T$ has been constructed to be continuous but violate $\sigma$, i.e.,

$$
\sup _{j \rightarrow \infty} \frac{\left\|T e_{n_{j}}\right\|_{k}}{\left|e_{n_{j}}\right|_{\sigma(k)}}=\lim _{j \rightarrow \infty} \exp \left\{(\sigma(k+1)-\sigma(k)) \beta_{n_{j}}\right\}=\infty .
$$

From (8) it follows that there is a subsequence of $\left(\beta_{n_{j}}\right)$ such that there are no repetitions in $\left(q_{j}^{k}\right)$. So $T$ restricted to this coordinate subspace of $\Lambda_{\infty}\left(\beta_{n_{j}}\right)$ is an embedding.

If for a given $\alpha$ there is some $\beta$ such that Theorem 2.1(iii) is satisfied, then in fact this $\beta$ can be chosen to be $\alpha$. Just set $\alpha_{n_{j}}=\alpha_{n(j, 1)}$; then

$$
\lim _{j \rightarrow \infty} \frac{\alpha_{m(j, k)}}{\alpha_{n_{j}}}=\frac{r_{k}}{r_{1}}
$$

for every $k \in \mathbf{N}$ and $\lim _{k \rightarrow \infty} r_{k} / r_{1}=\infty$. From Theorem 2.1 we obtain

2.2. THEOREM. $\Lambda_{1}(\alpha)$ contains a subspace isomorphic to an infinite type power series space if and only if there is an infinite subset $M \subset \mathbf{N}$ such that $\operatorname{LIM}\left\{\alpha_{m} / \alpha_{n} \mid m \in \mathbf{N}\right.$, $n \in M_{1}$ \} is unbounded for every infinite $M_{1} \subset M$. 
An equivalent formulation of this theorem is the following corrected version of Kashirin's theorem [6].

2.3. THEOREM. $\Lambda_{1}(\alpha)$ contains a subspace isomorphic to an infinite type power series space if and only if there is a subsequence $\left(\alpha_{n}\right)$ of $\alpha$, real numbers $M(k)>k, k \in \mathbf{N}$, and indices $m(k, j)$ and $j_{k}$ such that

$$
k \leqslant \alpha_{m(k, j)} / \alpha_{n_{j}} \leqslant M(k) \text { for } j \geqslant j_{k}, k \in \mathbf{N} .
$$

EXAMPLES. 1. Define an exponent sequence $\left(\alpha_{n}\right)$ such that $\alpha_{1}=1$ and

$$
\alpha_{n+1} / \alpha_{n}=(1,2,1,2,3,1,2,3,4,1,2, \ldots) .
$$

For the complete definition see [2], where the following problem was posed: does the space $\Lambda_{1}(\alpha)$ contain infinite type subspaces? In view of Theorems 2.1 or 2.3 , it clearly does. Choose $\left(\alpha_{n_{j}}\right)$ such that $\alpha_{n_{j}+1} / \alpha_{n_{j}}=1$. Then

$$
\lim _{j \rightarrow \infty} \alpha_{n_{j}+k} / \alpha_{n_{j}}=k !
$$

So (9) is satisfied.

2. Let $\alpha$ be an exponent sequence such that $\alpha_{1}=1$ and

$$
\alpha_{n+1} / \alpha_{n}=(1,2,1,3,2,3,1,4,2,4,3,4,1,5,2, \ldots) .
$$

Then $\operatorname{LIM}\{\alpha / \alpha\}=\mathbf{N}$, so it is unbounded, but $\alpha$ does not have a subsequence such that (9) would be satisfied.

Suppose $\left(\alpha_{n_{j}}\right)$ were such a subsequence. Let $k \in \mathbf{N}$ be a limit point and $m(k, j)$ indices such that

$$
\lim _{j \rightarrow \infty} \frac{\alpha_{m(k, j)}}{\alpha_{n_{j}}}=k
$$

Then for $j$ sufficiently large we have $\alpha_{m(k, j)} / \alpha_{n_{j}}=k$, so $m(k, j)=n_{j}+1$ by the definition of $\alpha$. Now let $l>k$ be a limit point and $m(l, j)$ the indices such that

$$
\lim _{j \rightarrow \infty} \frac{\alpha_{m(l, j)}}{\alpha_{n_{j}}}=l \text {. }
$$

Then $m(l, j) \geqslant n_{j}+2$ for $j$ sufficiently large and

$$
\lim _{j \rightarrow \infty} \frac{\alpha_{m(l, j)}}{\alpha_{n_{j}}} \geqslant \lim _{j \rightarrow \infty} \frac{\alpha_{n_{j}+2}}{\alpha_{n_{j}}}=\infty .
$$

Thus, although there exist noncompact operators from infinite type power series spaces to $\Lambda_{1}(\alpha)$, none of them can be an embedding.

3. Finite type quotients of an infinite type power series space. In this section we show how the existence of noncompact mappings from $\Lambda_{\infty}(\beta)$ to $\Lambda_{1}(\alpha)$ is related to the existence of quotient mappings, i.e., continuous, open and surjective mappings from $\Lambda_{\infty}(\beta)$ to a coordinate subspace of $\Lambda_{1}(\alpha)$. We prove the following.

3.1. THEOREM. The following conditions are equivalent.

(i) A coordinate subspace of $\Lambda_{1}(\alpha)$ is isomorphic to a quotient space of $\Lambda_{\infty}(\beta)$.

(ii) There is an infinite subset $M \subset \mathbf{N}$ such that $\operatorname{LIM}\left\{\alpha_{m} / \beta_{n} \mid m \in M_{1}, n \in \mathbf{N}\right\}$ is unbounded for every infinite $M_{1} \subset M$. 
(iii) There is a subsequence $\left(\alpha_{m_{j}}\right)$ of $\alpha$, a sequence of positive numbers $\left(r_{k}\right)$ with $\lim _{k \rightarrow \infty} r_{k}=\infty$ and indices $n(j, k)$ such that $\lim _{j \rightarrow \infty} \alpha_{m_{j}} / \beta_{n(j, k)}=r_{k}$ for every $k \in \mathbf{N}$.

Proof. As in the proof of Theorem 2.1 the implication (i) $\Rightarrow$ (ii) follows from Proposition 1.5. Assuming (ii), we obtain a subsequence $\left(\alpha_{m}\right)$ satisfying (iii) in a manner analogous to the corresponding proof in Theorem 2.1. What remains to show is that under (iii) we can construct a quotient mapping from $\Lambda_{\infty}(\beta)$ onto a coordinate subspace of $\Lambda_{1}(\alpha)$. Given the sequence $\left(r_{k}\right)$ of limit points we can find a strictly increasing sequence $\left(\nu_{k}\right)$ of indices with $\lim _{k \rightarrow \infty} \nu_{k}=\infty$ such that there is at least one element of the sequence $\left(r_{k}\right)$ on each interval $\left(\nu_{k-1} \nu_{k} /\left(\nu_{k}-\nu_{k-1}\right)\right.$, $\left.\nu_{k} \nu_{k+1} /\left(\nu_{k+1}-\nu_{k}\right)\right)$. So for $j$ sufficiently large, say $j \geqslant j_{k}$, there exist indices $p_{j}^{k}$ such that

$$
\frac{\nu_{k-1} \nu_{k}}{\nu_{k}-\nu_{k-1}}<\frac{\alpha_{j}}{\beta_{p_{j}^{k}}}<\frac{\nu_{k} \nu_{k+1}}{\nu_{k+1}-\nu_{k}} .
$$

Since for any fixed $j$ only a finite number of indices $p_{j}^{k}$ are chosen, we may assume, passing to a subsequence of $\left(\alpha_{j}\right)$ if necessary, that all the indices $p_{j}^{k}$ are distinct. Set

$$
t_{p_{j}^{k}}=\exp \left\{k \beta_{p_{j}^{k}}+\alpha_{j} / \nu_{k}\right\}
$$

and define for the coordinate basis vectors $e_{i} \in \Lambda_{\infty}(\beta)$ and $e_{j} \in \Lambda_{1}(\alpha)$ :

$$
T e_{i}= \begin{cases}t_{p_{j}^{k}} e_{j} & \text { for } i=p_{j}^{k} \\ 0 & \text { otherwise }\end{cases}
$$

Then

$$
\left\|T e_{p_{j}^{k}}\right\|_{\nu_{k}}=\left|e_{p_{j}^{k}}\right|_{k} .
$$

Moreover, using (10) and induction we can prove (analogously to the proof of Proposition 1.6) that

$$
\left\|T e_{p_{j}^{k}}\right\|_{\nu_{l}} \leqslant\left|e_{p_{j}^{k}}\right|_{l}
$$

for every $l \in \mathbf{N}$. So $T$ extends to a continuous linear mapping from $\Lambda_{\infty}(\beta)$ to $\Lambda_{1}(\alpha)$. It also follows from (11) that for every coordinate basis vector $e_{j}$ of $\Lambda_{1}(\alpha)$, where $j_{k} \leqslant j<j_{k+1}$, and for every $l=1,2, \ldots, k$, there is a vector $x_{j}^{l} \in \Lambda_{\infty}(\beta)$,

$$
x_{j}^{l}=\left(t_{p_{j}^{\prime}}\right)^{-1} e_{p_{j}^{\prime}}, \quad T x_{j}^{l}=e_{j},
$$

such that

$$
\left|x_{j}\right|_{l} \leqslant\left\|e_{j}\right\|_{\nu_{i}}
$$

So $T$ is open and onto and hence a quotient mapping from $\Lambda_{\infty}(\beta)$ to $\Lambda_{1}(\alpha)$.

Similarly as in the case of subspaces, we see that if conditions (ii) or (iii) in the previous theorem are satisfied for some $\alpha$, then, in fact, $\alpha$ can be replaced by $\beta$. So we have

3.2. THEOREM. $\Lambda_{\infty}(\beta)$ has a quotient space isomorphic to a finite type power series space if and only if there is an infinite subset $M \subset \mathbf{N}$ such that $\operatorname{LIM}\left\{\beta_{m} / \beta_{n} \mid m \in M_{1}\right.$, $n \in \mathbf{N}\}$ is unbounded for every infinite $M_{1} \subset M$. 
4. Splitting short exact sequences. Let $E, F$ and $X$ be Fréchet spaces such that there is an isomorphism $i$ from $E$ to a closed subspace $i(E)$ of $X$ and $F$ is isomorphic to the quotient space $X / i(E)$. Then we say that these spaces form a short exact sequence

$$
0 \rightarrow E \stackrel{i}{\rightarrow} X \stackrel{q}{\rightarrow} F \rightarrow 0,
$$

where $q$ is an open operator onto $F$ with $\operatorname{Ker} q=\operatorname{Im} i$. We say that the sequence splits if there exists a continuous right inverse of $q$ or, equivalently, a continuous left inverse of $i$, which means $E$ and $F$ are isomorphic to complementary subspaces of $X$.

A method of constructing short exact sequences of nuclear Fréchet spaces was given in [10]. Also, in [10, Theorem 4.1], a condition was derived under which every short exact sequence

$$
0 \rightarrow K(b) \rightarrow X \rightarrow K(a) \rightarrow 0
$$

splits.

By nuclearity this condition can be given in the following equivalent form: For every increasing function $\sigma: \mathbf{N} \rightarrow \mathbf{N}$ there is a function $\rho: \mathbf{N} \rightarrow \mathbf{N}$ such that for every pair $p, q, p>q$, we have

$$
\max _{q \leqslant r<p} \frac{a_{m}^{\sigma(r)}}{b_{n}^{r}} \leqslant \max \left\{\frac{a_{m}^{\rho(q)}}{b_{n}^{q}}, \frac{a_{m}^{\rho(p)}}{b_{n}^{p}}\right\}
$$

for every $m$ and $n$ sufficiently large.

If in the short exact sequence (12) the Köthe space $K(a)$ is a power series space of infinite type, the sequence splits (see e.g. [15]). This fact can also be verified using (13). Examples of nonsplitting sequences (12) can be given where $K(a)$ is a finite type power series space and $K(b)$ is a power series space of finite type [16, 10] or infinite type $[12,10]$. The following theorem completely describes the situation.

4.1. THEOREM. The following conditions are equivalent.

(i) Every short exact sequence $0 \rightarrow \Lambda_{1}(\beta) \rightarrow X \rightarrow \Lambda_{1}(\alpha) \rightarrow 0$ splits.

(ii) Every short exact sequence $0 \rightarrow \Lambda_{\infty}(\beta) \rightarrow X \rightarrow \Lambda_{1}(\alpha) \rightarrow 0$ splits.

(iii) $\operatorname{LIM}\{\alpha / \beta\}$ is bounded.

Proof. To show (iii) $\Rightarrow$ (ii) and (iii) $\Rightarrow$ (i) we make use of (13). For $K(a)=\Lambda_{1}(\alpha)$ and $K(b)=\Lambda_{\infty}(\beta)$ the inequality in (13) is equivalent to

$$
\min _{q \leqslant r<p}\left(\alpha_{m} / \sigma(r)+r \beta_{n}\right) \geqslant \min _{r=q, p}\left(\alpha_{m} / \rho(r)+r \beta_{n}\right) .
$$

So it is enough to show that the minimum of

$$
\frac{\alpha_{m}}{\sigma(r)}+r \beta_{n}=\beta_{n}\left(\frac{\alpha_{m}}{\beta_{n}} \cdot \frac{1}{\sigma(r)}+r\right)
$$

as $r$ varies is attained either for $r=p$ or $r=q$ for almost all $m$ and $n$.

Let $B$ be a strict upper bound for the finite limit points of the ratios $\alpha_{m} / \beta_{n}$. We may assume $\sigma(1)>2 B$. Fix $p$ and $q, p>q$. Then for almost all $m$ and $n$ either

$$
\frac{\alpha_{m}}{\beta_{n}}<B \quad \text { or } \quad \frac{\alpha_{m}}{\beta_{n}}>\max _{q \leqslant r<p} \frac{\sigma(r+1) \sigma(r)}{\sigma(r+1)-\sigma(r) .}
$$


In the first case the minimum of (14) is attained for $r=q$, since

$$
0<\frac{\alpha_{m}}{\beta_{n}} \cdot \frac{1}{\sigma(r)} \leqslant B \cdot \frac{1}{\sigma(1)}<\frac{1}{2} .
$$

In the second case the minimum of (14) is attained for $r=p$, since

$$
\frac{\alpha_{m}}{\beta_{n}} \frac{1}{\sigma(r+1)}+r+1-\frac{\alpha_{m}}{\beta_{n}} \cdot \frac{1}{\sigma(r)}-r=-\frac{\sigma(r+1)-\sigma(r)}{\sigma(r) \sigma(r+1)} \cdot \frac{\alpha_{m}}{\beta_{n}}+1 \leqslant 0
$$

for every $r, q \leqslant r<p$.

The proof of (iii) $\Rightarrow$ (i) is similar. To prove the converse implications we refer to $[10, \S 3]$, where examples of nonsplitting sequences $0 \rightarrow \Lambda_{1}(\beta) \rightarrow X \rightarrow \Lambda_{1}(\alpha) \rightarrow 0$ and $0 \rightarrow \Lambda_{\infty}(\beta) \rightarrow X \rightarrow \Lambda_{1}(\alpha) \rightarrow 0$ were constructed. To carry through these constructions it is sufficient that for every increasing sequence $\left(t_{i}\right)$ of positive numbers with $\lim _{i \rightarrow \infty} t_{i}=\infty$, there are arbitrarily large indices $i$ for which there exist arbitrarily large indices $m$ with the nonempty set

$$
\left\{n \in \mathbf{N} \mid \alpha_{m} / t_{i+1}<\beta_{n} \leqslant \alpha_{m} / t_{i}\right\}=\left\{n \in \mathbf{N} \mid t_{i} \leqslant \alpha_{m} / \beta_{n}<t_{i+1}\right\} .
$$

But this is nothing but the negation of (iii). Let us remark that in [10, Lemma 3.1] it was shown that this condition is satified for any $\alpha$ if $\sup \beta_{n+1} / \beta_{n}<\infty$.

5. Products of power series spaces. A Schauder basis $\left(x_{n}\right)$ of a nuclear Fréchet space $E$ is regular if there is a fundamental sequence of continuous seminorms $\|\cdot\|_{k}$, $k \in \mathbf{N}$, in $E$ such that

$$
\frac{\left\|x_{n}\right\|_{k}}{\left\|x_{n}\right\|_{k+1}} \geqslant \frac{\left\|x_{n+1}\right\|_{k}}{\left\|x_{n+1}\right\|_{k+1}}
$$

for all $n, k \in \mathbf{N}$.

In [1] a necessary and sufficient condition on $\alpha$ and $\beta$ was given under which $\Lambda_{1}(\alpha) \times \Lambda_{\infty}(\beta)$ has a regular basis. As suggested to the author by Dubinsky, this condition is satisfied if and only if $\operatorname{LIM}\{\alpha / \beta\}$ is bounded.

5.1. Lemma. $\operatorname{LIM}\{\alpha / \beta\}$ is bounded if and only if there exist strictly increasing sequences of indices $\left(m_{i}\right)$ and $\left(n_{i}\right)$ such that

$$
\sup _{i} \frac{\alpha_{m_{i+1}}}{\beta_{n_{i}+1}}<\infty \text { and } \lim _{i \rightarrow \infty} \frac{\alpha_{m_{i}+1}}{\beta_{n_{i}}}=\infty \text {. }
$$

Proof. Let $B>\alpha_{1} / \beta_{1}$ be a strict upper bound for the set of the finite limit points of $\left\{\alpha_{m} / \beta_{n} \mid m, n \in \mathbf{N}\right\}$. We choose

$$
m_{1}=\max \left\{m \mid \alpha_{m} \leqslant B \beta_{1}\right\}
$$

then $\alpha_{m_{1}+1} / \beta_{1}>B$; and we set

$$
n_{1}=\max \left\{n \mid B \beta_{n}<\alpha_{m_{1}+1}\right\} .
$$

Suppose $m_{i}$ and $n_{i}$ have been chosen in such a way that

$$
m_{i}=\max \left\{m \mid \alpha_{m} \leqslant B \beta_{n_{i-1}+1}\right\}, \quad n_{i}=\max \left\{n \mid B \beta_{n}<\alpha_{m_{i}+1}\right\} ;
$$


Then $B \beta_{n_{i}+1} \geqslant \alpha_{m_{i}+1}$; so choose

$$
m_{i+1}=\max \left\{m \mid \alpha_{m} \leqslant B \beta_{n_{i}+1}\right\} \geqslant m_{i}+1 .
$$

Since $\alpha_{m_{i+1}+1}>B \beta_{n_{i}+1}$, we can take

$$
n_{i+1}=\max \left\{n \mid B \beta_{n}<\alpha_{m_{i, 1}+1}\right\} \geqslant n_{i}+1 .
$$

So, by induction, we construct subsequences $\left(m_{i}\right)$ and $\left(n_{i}\right)$ of $\mathbf{N}$ such that

$$
\alpha_{m_{i+1}} / \beta_{n_{i}+1} \leqslant B \text { and } \alpha_{m_{i}+1} / \beta_{n_{i}}>B, \quad i \in \mathbf{N} .
$$

Since the finite limit points of $\left\{\alpha_{m} / \beta_{n} \mid m, n \in \mathbf{N}\right\}$ are strictly less than $B$, we have

$$
\sup _{i \in \mathbf{N}} \frac{\alpha_{m_{i+1}}}{\beta_{n_{i}+1}} \leqslant B \text { and } \quad \lim _{i \rightarrow \infty} \frac{\alpha_{m_{i}+1}}{\beta_{n_{i}}}=\infty \text {. }
$$

Suppose now that such subsequences $\left(m_{i}\right)$ and $\left(n_{i}\right)$ exist and denote

$$
B=\sup _{i} \frac{\alpha_{m_{i+1}}}{\beta_{n_{i}+1}}<\infty \text {. }
$$

We show that $B$ is an upper bound for the finite limit points. Let $p>B$ be a limit point of $\left\{\alpha_{m} / \beta_{n} \mid m, n \in \mathbf{N}\right\}$, i. e.,

$$
p=\lim _{k \rightarrow \infty} \frac{\alpha_{\mu_{k}}}{\beta_{\nu_{k}}} .
$$

When $\alpha_{\mu_{k}} / \beta_{\nu_{k}}>B$, i.e., $k$ is sufficiently large, and $m_{i}<\mu_{k} \leqslant m_{i+1}$, then $\nu_{k} \leqslant n_{i}$. Consequently,

$$
\alpha_{\mu_{k}} / \beta_{v_{k}} \geqslant \alpha_{m_{i}+1} / \beta_{n_{i}} .
$$

Since $\lim _{i \rightarrow \infty}\left(\alpha_{m_{i}+1} / \beta_{n_{i}}\right)=\infty$, the limit point $p$ is infinity.

Summarizing some of the results from [1] we get

5.2. THEOREM. The following conditions are equivalent.

(i) $\Lambda_{1}(\alpha) \times \Lambda_{\infty}(\beta)$ has a regular basis.

(ii) $\Lambda_{1}(\alpha) \otimes \Lambda_{\infty}(\beta)$ has a regular basis.

(iii) $\Lambda_{1}(\alpha) \otimes \Lambda_{\infty}(\beta) \simeq \Lambda_{1}(\tilde{\alpha}) \times \Lambda_{\infty}(\tilde{\beta})$, where $\tilde{\alpha}$ and $\tilde{\beta}$ can be obtained from $\alpha$ and $\beta$ by repeating their terms.

(iv) $\operatorname{LIM}\{\alpha / \beta\}$ is bounded.

For the proofs we refer to [1]. However, a short alternative proof can now be given to the equivalence (iii) $\Leftrightarrow$ (iv): The tensor product $\Lambda_{1}(\alpha) \otimes \Lambda_{\infty}(\beta)$ can be presented as a Köthe space $K(a)$, where the Köthe matrix $a=\left(a_{n}^{k}\right)$ is

$$
a_{n}^{k}=\exp \left\{\left(-1 / k+k \lambda_{n}\right) \gamma_{n}\right\} \text {. }
$$

Here

$$
\lambda_{n}=\beta_{\nu} /\left(\alpha_{\mu}+\beta_{\nu}\right), \quad \gamma_{n}=\alpha_{\mu}+\beta_{\nu}, \quad \text { for } n=\sigma(\mu, \nu),
$$

and $\sigma$ is any bijection $\mathbf{N} \times \mathbf{N} \rightarrow \mathbf{N}$ (see [19]). For a subsequence $\left(n_{j}\right)$ the corresponding coordinate subspace $K\left(a_{n_{1}}^{p}\right)$ is isomorphic to an infinite (resp. finite) type power series space if and only if $\inf _{j \in \mathbf{N}} \lambda_{n_{j}}>0\left(\lim _{j \rightarrow \infty} \lambda_{n_{j}}=0\right)$. Now $\operatorname{LIM}\{\alpha / \beta\}$ is 
bounded exactly when $\mathbf{N} \times \mathbf{N}$ can be split into two disjoint sets $I$ and $J$ such that

$$
\lim _{(\mu, \nu) \in I} \frac{\alpha_{\mu}}{\beta_{\nu}}=\infty \quad \text { and } \quad \sup _{(\mu, \nu) \in J} \frac{\alpha_{\mu}}{\beta_{\nu}}<\infty .
$$

But this is the case when

$$
K(a)=K(a)_{\sigma(I)} \oplus K(a)_{\sigma(J)},
$$

where

$$
K(a)_{\sigma(I)}=K\left(\left(a_{\sigma(\mu, \nu)}^{k}\right)_{(\mu, \nu) \in I}\right) \simeq \Lambda_{1}(\tilde{\alpha})
$$

and

$$
K(a)_{\sigma(J)}=K\left(\left(a_{\sigma(\mu, \nu)}^{k}\right)_{(\mu, \nu) \in J}\right) \simeq \Lambda_{\infty}(\tilde{\beta})
$$

for some exponent sequences $\tilde{\alpha}$ and $\tilde{\beta}$. It is not hard to see that then $\tilde{\alpha}$ and $\tilde{\beta}$ are equivalent to exponent sequences obtained from $\alpha$ and $\beta$ by repeating their terms finitely many times.

Taking the equivalence of (iii) and (iv) into consideration we see that Theorem 1.8 remains valid if the cartesian product $\Lambda_{1}(\alpha) \times \Lambda_{\infty}(\beta)$ is replaced by the tensor product $\Lambda_{1}(\alpha) \otimes \Lambda_{\infty}(\beta)$.

\section{REFERENCES}

1. L. Crone, E. Dubinsky and W. B. Robinson, Regular bases in products of power series spaces, J. Funct. Anal. 24 (1977), 211-222.

2. N. DeGrande-DeKimpe and W. B. Robinson, Compact maps and embeddings from an infinite type power series space to a finite type power series space, J. Reine Angew. Math. 293 / 294 (1977), 51-61.

3. M. M. Dragilev, Riesz classes and multiple regular bases, Teor. Funkcii Funkcional Anal. i Priložen. 15 (1972), 55-78. (Russian)

4. E. Dubinsky, Infinite type power series subspaces of finite type power series spaces, Israel J. Math. 15 (1973), 257-281.

5. , Structure of nuclear Fréchet spaces, Lecture Notes in Math., vol. 720, Springer-Verlag, Berlin and New York, 1979.

6. V. V. Kashirin, Subspaces of a finite center of an absolute Riesz scale which are isomorphic to an infinite center, Siberian Math. J. 16 (1975), no. 4, 863-865. (Russian)

7. __ Isomorphic embeddings to some generalized power series spaces, preprint, Warsaw, 1979.

8. Comparison of linear dimensions of some generalized power series spaces, Bull. Acad. Polon. Sci. Sér. Sci. Math. 27 (1980), 567-569.

9. , Isomorphic embeddings of some generalized power series spaces, Studia Math. 71 (1981), 169-178.

10. T. Ketonen and K. Nyberg, Twisted sums of nuclear Fréchet spaces, Ann. Acad. Sci. Fenn. Ser. A I Math. 7 (1982), 323-335.

11. B. S. Mitjagin, Equivalence of bases in Hilbert scales, Studia Math. 37 (1971), 111-137. (Russian)

12. B. S. Mitjagin and G. M. Henkin, Linear problems of complex analysis, Russian Math. Surveys 26 (1971), 99-164.

13. Z. Nurlu, On basic sequences in some Köthe spaces and existence of non-compact operators, Thesis, Potsdam, 1981.

14. On pairs of Köthe spaces between which all operators are compact (preprint).

15. D. Vogt and M. J. Wagner, Charakterisierung der Unterräume und Quotientenräume der nuklearen stabilen Potenzreihenräume von unendlichem Typ, Studia Math. 70 (1981), 63-80. 
16. D. Vogt, Eine Charakterisierung der Potenzreihenräume von endlichem Typ und ihre Folgerungen, Manuscripta Math. 37 (1982), 269-301.

17. __ Frécheträume zwischendenen jede stetige lineare Abbildung beschränkt ist, J. Reine Angew. Math. (to appear).

18. V. P. Zaharjuta, On the isomorphism of cartesian products of locally convex spaces, Studia Math. 46 (1973), 201-221.

19. On the isomorphism and quasiequivalence of bases in Köthe sequence spaces, Seventh Winter School Math. Programming, (Drogobych, 1974), 101-126. (Russian)

Department of Mathematics, University of Helsinki, Hallituskatu 15, SF - 00100 Helsinki 10. FINLAND

Current address: Av. des Alouettes 15, B-1640 Rhode-St. Genèse, Belgium 